Revue de droit comparé du travail et de la sécurité sociale

2| 2018

Harcèlement moral au travail en jurisprudence

comparée

\title{
Le harcèlement au travail dans la jurisprudence française : Délimitation du périmètre et débat sur la responsabilité de l'employeur
}

Loïc Lerouge

\section{OpenEdition}

Journals

Édition électronique

URL : https://journals.openedition.org/rdctss/1944

DOI : $10.4000 /$ rdctss. 1944

ISSN : 2262-9815

Éditeur

Centre de droit comparé du travail et de la sécurité sociale

Édition imprimée

Date de publication : 1 juin 2018

Pagination : $30-39$

ISSN : 2117-4350

Référence électronique

Loïc Lerouge, « Le harcèlement au travail dans la jurisprudence française : Délimitation du périmètre et débat sur la responsabilité de l'employeur », Revue de droit comparé du travail et de la sécurité sociale [En ligne], 2 | 2018, mis en ligne le 01 novembre 2021, consulté le 13 novembre 2021. URL : http:// journals.openedition.org/rdctss/1944; DOI : https://doi.org/10.4000/rdctss.1944

\section{(c) $(1)$}

Revue de droit comparé du travail et de la sécurité sociale est mise à disposition selon les termes de la Licence Creative Commons Attribution - Pas d'Utilisation Commerciale - Pas de Modification 4.0 International. 


\section{LE HARCÈLEMENT AU TRAVAIL \\ DANS LA JURISPRUDENCE FRANÇAISE : DÉLIMITATION DU PÉRIMÈTRE ET DÉBAT SUR LA RESPONSABILITÉ DE L'EMPLOYEUR}

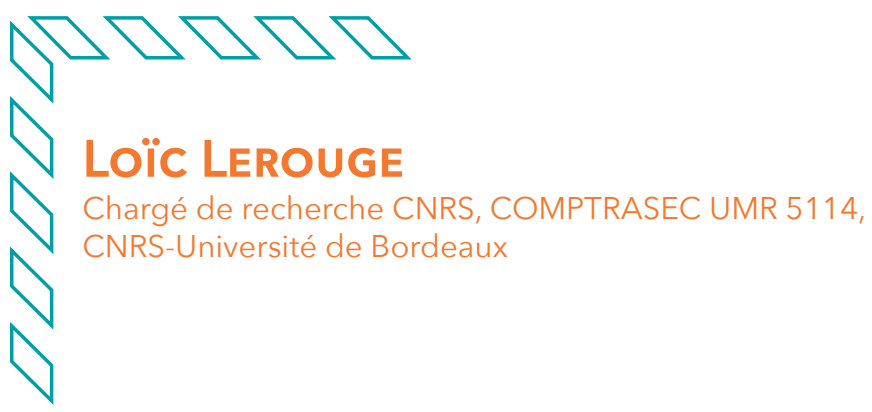

L

e harcèlement moral au travail demeure depuis autant de temps que les relations de travail elles-mêmes existent. Le professeur Christophe Dejours rappelle par exemple que le harcèlement moral existait sur les chaines de montage dans les années 1970 avec les " petits chefs " ${ }^{1}$. La question a cependant fait véritablement irruption dans le débat public à travers la publication de l'ouvrage du docteur Marie-France Hirigoyen sur «Le harcèlement moral. La violence perverse au quotidien $»^{2}$ qui a marqué un tournant. Le terme de "harcèlement moral » a permis de désigner une forme de violence au travail qui se traduit par des agissements répétés entrainant une dégradation des conditions de travail à l'encontre d'un travailleur.

De nombreuses associations de lutte contre le harcèlement moral ont été créées au niveau national et régional ${ }^{3}$, deux propositions de loi ont été déposées (Georges Hage en 1999 côté Sénat et Roland Muzeau en 2000 côté Assemblée nationale). En 2001, Le Conseil Économique et Social et Environnemental (CESE) a publié un avis incluant une proposition de définition, le rapporteur étant le

1 V. entretien avec Christophe Dejours, "Harcèlement et santé au travail », https://www.youtube.com/watch?v=LOrycUEJgRM, mise en ligne le 20 octobre 2017.

2 M.-F. Hirigoyen, Le harcèlement moral. La violence perverse au quotidien, Éd. La Découverte et Syros, Coll. Pocket, 1998, 252 p.

3 Par exemple Mots pour Maux au travail, Association nationale des victimes de harcèlement psychologique au travail (ANVHPT), Harcèlement Moral Stop, Association contre le harcèlement professionnel, Harcèlement association de réflexion et de soutien, Association de défense contre le harcèlement moral, ... 
professeur Michel Debout ${ }^{4}$. Ces débats sur le harcèlement moral au travail ont permis d'alimenter les travaux qui ont abouti à un chapitre qui lui a été dédié au sein de la loi de modernisation sociale du 17 janvier 2002.

Le système français présente la particularité d'avoir décliné une même définition juridique en droit du travail, en droit pénal et en droit de la fonction publique ${ }^{5}$. Selon l'article L. 1152-1 du Code du travail (reproduit à l'article 233-2 du Code pénal et à l'article 6 quinquiès du 13 juillet 1983 portant droits et obligations des fonctionnaires), "Aucun salarié [ou fonctionnaire] ne doit subir les agissements répétés de harcèlement moral qui ont pour objet ou pour effet une dégradation de ses conditions de travail susceptible de porter atteinte à ses droits et à sa dignité, d'altérer sa santé physique ou mentale ou de compromettre son avenir professionnel ". Trois critères cumulatifs doivent être réunis pour qualifier le harcèlement moral : les agissements litigieux doivent être "répétés » (un acte isolé ne peut donc pas conduire à sa qualification); ils ont pour objet ou pour effet une dégradation des conditions de travail de la victime; ils doivent être susceptibles de porter atteinte à sa santé physique ou mentale ou de compromettre son avenir professionnel. L'article 233-2 du Code pénal punit l'incrimination de harcèlement moral au travail de deux ans d'emprisonnement et de 30000 euros d'amende.

Outre une définition en droit, le système français a introduit des dispositifs juridiques pour lutter contre le harcèlement moral au travail. Le premier est de reconnaître la nullité de la rupture du contrat de travail qui méconnaît la définition du harcèlement moral (art. L. 1152-3 CT). Selon l'article L. 1152-4 du Code du travail, l'employeur est tenu de prendre les dispositions nécessaires pour prévenir les agissements de harcèlement moral et doit prévenir les atteintes à la santé physique et mentale au travail (art. L. 4121-1 CT) ${ }^{6}$. II doit également inscrire dans le règlement intérieur les dispositions relatives à l'interdiction de toute pratique de harcèlement moral et sexuel (art. L. 1321-2 CT). Du côté salarié, l'article L. 1152-5 précise que dès lors qu'il est l'auteur de tels agissements, il est passible de sanctions disciplinaires. Le salarié, la personne en formation ou en stage qui dénonce des agissements de harcèlement moral est protégé contre des mesures discriminatoires (en matière de rémunération, de formation, de reclassement, d'affectation, de qualification, de classification, de promotion professionnelle, de mutation ou de renouvellement de contrat ; art. L. 1152-2 CT). Une procédure de médiation a été instituée de manière inédite dans le Code du travail pour toute personne s'estimant victime de harcèlement moral (L. $1152-6$ CT).

4 http://www.lecese.fr/travaux-publies/le-harcelement-moral-au-travail

5 Notons que le régime juridique du harcèlement moral au travail est différent en France entre le secteur privé et la fonction publique, voir L. Lerouge L., «Les différences de traitement juridique du harcèlement moral dans le secteur privé et la fonction publique : des rapprochements possibles?», Droit social, 2012, p. 483-490.

6 Pour lutter contre le harcèlement moral au travail, la loi du 17 janvier 2002 a également introduit la notion de "santé physique et mentale " dans les compétences du Comité d'hygiène, de sécurité et des conditions de travail (CHSCT est regroupé désormais avec les autres institutions représentatives du personnel dans le Comité Social et Économique depuis les ordonnances sur la réforme du droit du travail du 22 septembre 2017), du médecin du travail et dans le droit d'alerte du personnel 
Le législateur a aussi donné des moyens aux représentants du personnel et syndicaux. Ainsi le délégué du personnel possède un droit d'alerte en matière d'atteinte aux droits des personnes, à leur santé physique et mentale ou aux libertés individuelles résultant de faits de harcèlement sexuel ou moral ou de toute mesure discriminatoire et qui ne serait pas justifiée par la nature de la tâche à accomplir, ni proportionnée au but recherché (art. L. 2313-2 CT). À un niveau plus collectif et sur le fondement de l'article L. 4612-3 du Code du travail, le Comité d'hygiène, de sécurité et des conditions de travail (CHSCT) peut proposer notamment des actions de prévention du harcèlement moral, du harcèlement sexuel et des agissements sexiste. Le refus de l'employeur de les appliquer doit être motivé. En vertu des ordonnances du 22 septembre portant réforme du droit du travail, ces institutions représentatives du personnel seront cependant regroupées en une seule appelée Comité social et économique (CSE). Enfin, les organisations syndicales dans l'entreprise peuvent aussi se substituer à la victime de harcèlement moral avec son accord pour exercer une action en justice en sa faveur (action en substitution, article L. 1154-2 CT).

Dans une situation de harcèlement moral au travail, le salarié est considéré comme la partie faible au contrat de travail. Pour cette raison, comme en matière de discrimination, et de manière à en faciliter la démonstration, le législateur a procédé à un aménagement de la charge de la preuve. Ainsi, " le candidat à un emploi, à un stage ou à une période de formation en entreprise ou le salarié établit des faits qui permettent de présumer l'existence d'un harcèlement. Au vu de ces éléments, il incombe à la partie défenderesse de prouver que ces agissements ne sont pas constitutifs d'un tel harcèlement et que sa décision est justifiée par des éléments objectifs étrangers à tout harcèlement. Le juge forme sa conviction après avoir ordonné, en cas de besoin, toutes les mesures d'instruction qu'il estime utiles » (art. L. 1154-1 CT).

Au contentieux, les sanctions qui accompagnent le harcèlement peuvent être diverses en fonction de son origine. La première des sanctions est plus symbolique que juridique et s'exprime dans la reconnaissance même du harcèlement et de la condamnation de l'employeur. Lorsque le harcèlement trouve sa source dans une décision de l'employeur (par exemple une sanction disciplinaire), la victime pourra en obtenir l'annulation, avec toutes les conséquences sur sa situation de travail. Le salarié qui a été harcelé devra retrouver son état d'avant la sanction disciplinaire. Toutefois, pour obtenir des dommages et intérêts, il s'agira de prouver les dommages causés par la situation de harcèlement moral à l'égard de la victime. Le principe de l'aménagement de la charge de la preuve n'existe pas ici ; la preuve doit être rapportée par le demandeur. L'évaluation du préjudice psychologique n'est pas évidente, diverses interprétations sont possibles. Par exemple, la dépression de la victime est-elle réactionnelle à la situation de harcèlement ou bien d'autres facteurs l'expliquent-ils ? Le lien de causalité entre le dommage et le harcèlement moral doit être établi par la victime.

La définition juridique du harcèlement moral est de portée assez générale. Pour cette raison, et parce que le juge est seul compétent pour qualifier juridiquement le harcèlement moral, la définition a été précisée dans la jurisprudence. Le juge du travail français a mené un travail de précision considérable de la définition et de sa portée. En revanche, la jurisprudence administrative se veut plus restrictive. Les textes en la matière diffèrent du secteur privé ainsi que l'approche et la culture du travail. En raison de sa densité, il ne sera question dans cet article que d'effectuer une synthèse et une analyse de la jurisprudence française du harcèlement moral au travail dans le secteur privé. 
La Cour de cassation a en effet tenu un rôle important pour encadrer la qualification du harcèlement moral en droit du travail français à travers les décisions qu'elle a pu rendre. La jurisprudence en matière de harcèlement moral au travail est extrêmement consistante, l'objectif sera donc d'évoquer des décisions emblématiques qui ont façonné le droit du harcèlement moral en France pour en faire un panorama. Ces décisions se répartissent entre le contentieux du travail pour une grande partie (I), et le contentieux de sécurité sociale et pénal (II).

\section{I - HARCÈLEMENT MORAL ET JURISPRUDENCE EN DROIT DU TRAVAIL}

La définition de l'article L. 1152-1 du Code du travail portant définition du harcèlement moral au travail peut être interprétée de différentes manières selon la juridiction saisie, notamment au regard de son caractère général. Le contrôle de qualification est alors essentiel afin d'obtenir une interprétation par les juridictions du travail unifiée sur le territoire français (A). Ce travail de la Cour de cassation a été très important en la matière et s'est beaucoup articulé autour de l'obligation de sécurité de l'employeur (B).

\section{A - LE CONTRÔLE DE QUALIFICATION DU HARCÈLEMENT MORAL AU TRAVAIL PAR LES JUGES}

Le contrôle de qualification consiste pour le juge à vérifier, confirmer ou infirmer au regard du droit l'appréciation des faits, en l'occurrence, de la situation de harcèlement moral. Ce contrôle comprend pour ce genre de situation une importante casuistique qui n'est pas forcément habituelle au contrôle exercé par la Cour de cassation ${ }^{7}$. Le juge sera projeté dans une situation complexe où il devra fait la part entre les conditions normales de travail et celles qui ne le sont pas et qui seraient qualifiées de harcèlement moral.

Depuis l'entrée en vigueur des dispositifs de lutte contre le harcèlement moral au travail le 17 janvier 2002, le contentieux en la matière s'est considérablement multiplié. Après deux décisions qui rejetaient la qualification de harcèlement moral ${ }^{8}$, la Chambre sociale de la Cour de cassation a finalement décidé le 27 octobre 2004 d'abandonner cette qualification à l'appréciation souveraine des juges du fond'. Compte tenu de l'évolution du contentieux en matière de harcèlement au travail avec la multiplication des affaires, l'absence de contrôle de qualification de la Cour de cassation amenait des décisions diverses, pas forcément motivées comme il se doit ou qui se contredisent selon la juridiction du fond saisie. Un risque d'arbitraire pesait alors sur les décisions rendues par les juges du fond, par exemple concernant la qualification de la cause réelle et sérieuse de licenciement ou alors concernant les conséquences juridiques lourdes pesant sur l'auteur des faits selon que la qualification de harcèlement moral était retenue ou non.

7 Voir P. Adam, « Un contrôle, dans quel dessein ? ", Semaine Sociale Lamy, 29 septembre 2008, $n^{\circ} 1368$, propos recueillis par Françoise Champeaux, p. 12

8 Cass. soc. 9 mars 2004, n 02-473.422, inédit ; Cass. soc. 16 juin 2004, n 02-41.795, inédit, Lettre juridique Lexbase, $\mathrm{n}^{\circ} 141$, p. 2, note Ch. Radé.

9 Cass. soc. 27 octobre 2004, Semaine Sociale Lamy, n 1193, p. 11, RJS, 1/05, n 4, Droit Social, janvier 2005, p. 100, obs. C. Leroy-Loustaunau. 
En principe, la Cour de cassation n'a pas compétence de contrôler les faits, elle contrôle la conformité des décisions des juridictions du fond au droit. Jean-Yves Frouin, actuel Président de la Chambre sociale de la Cour de cassation, rappelait néanmoins que le juge de cassation contrôle la qualification juridique des faits « toutes les fois que le législateur donne, ou crée, une qualification légale à laquelle il attache des conséquences juridiques précises ». Autrement dit, la Cour de cassation s'assure que les juges du fond ont donné la qualification juridique exacte sans opérer un contrôle des constatations matérielles effectuées par les juges du fond ${ }^{10}$.

La chambre sociale de la Cour de cassation s'est toutefois réappropriée le 24 septembre 2008 le contrôle de qualification du harcèlement moral tant les questions associées à la définition légale du harcèlement moral énoncée par la loi de modernisation sociale ont rendu nécessaire la nécessité de l'intervention du juge du droit pour interpréter une notion encore nouvelle et complexe plutôt que de s'en remettre aux juges du fond ${ }^{11}$. La diversité des interprétations des juridictions du fond justifie aussi à elle seule cette "reprise en main » du contrôle de qualification par la Cour de cassation qui va ciseler le régime juridique du harcèlement moral au travail et faire le lien avec l'obligation de sécurité de l'employeur. La Cour de cassation a cependant évolué le 8 juin 2016 sur la question de la charge de la preuve du harcèlement moral au travail et l'appréciation des éléments invoqués par le salarié12. Elle redonne la main aux juges du fond qui vont apprécier souverainement si le salarié établit bien des faits qui permettent de présumer l'existence d'un harcèlement et si l'employeur prouve que les agissements invoqués sont étrangers à tout harcèlement.

\section{B - LE HARCÈLEMENT MORAL INDISSOCIABLE DE L'OBLIGATION DE SÉCURITÉ DE L'EMPLOYEUR}

Dans la construction du Code du travail français, la définition du harcèlement moral au travail est placée dans la partie I du Code du travail relative aux « relations individuelles du travail » à l'article 1152-1 du Code du travail alors que l'obligation de sécurité fait partie de la partie IV relative à la "santé et la sécurité au travail » au sein de l'article L. 4121-1. Des passerelles existent cependant entre les deux parties en matière de harcèlement moral au travail car la définition est dans la partie I tandis qu'un certain nombre de dispositifs imaginés pour lutter contre le harcèlement moral sont dans la partie IV. Ainsi, concernant l'obligation de sécurité à la charge de l'employeur, la loi de modernisation sociale a intégré la « santé physique et mentale » dans l'article L. 4121-1 du Code du travail dans l'idée de prendre en compte le harcèlement moral au sein cette obligation, ou le point 7 des principes généraux de prévention de l'article L. 4121-2 qui vise expressément le harcèlement moral (également sexuel et les agissements sexistes).

10 J.-Y. Frouin, «Sur le contrôle par la Cour de cassation de la qualification juridique de harcèlement moral », RJS, 10/05, p. 671.

$11 \mathrm{Ph}$. Waquet, "Un contrôle naturel et nécessaire », Semaine Sociale Lamy, 29 septembre 2008, $n^{\circ} 1368$, propos recueillis par Françoise Champeaux, p. 11 ; L. Lerouge, « Le contrôle de qualification du harcèlement moral au travail. À propos des quatre arrêts rendus par la Chambre sociale de la Cour de cassation le 24 septembre 2008 », Petites Affiches, n 1 et 2, janvier 2009, p. 7-14.

12 Cass. soc. 8 juin 2016, 14-13.418. 
Le 28 février 2002, la Chambre sociale de la Cour de cassation a décidé de qualifier l'obligation de sécurité dont est tenu l'employeur d'obligation de résultat ${ }^{13}$. Ce n'est toutefois que le 21 juin 2006 que le lien entre obligation de sécurité de résultat et harcèlement moral au travail a explicitement été reconnu par la Cour de cassation en faisant appel en se fondant sur les articles 1152-1 et 4121-1 du Code du travail, ce dernier interprété à la lumière de la Directive n 89/391 du 12 juin 1989 concernant la mise en œuvre de mesures visant à promouvoir l'amélioration de la sécurité et la santé des travailleurs: «l'employeur est tenu envers ses salariés d'une obligation de sécurité de résultat en matière de protection de la santé et de la sécurité des travailleurs dans l'entreprise, notamment en matière de harcèlement moral et que l'absence de faute de sa part ne peut l'exonérer de sa responsabilité ${ }^{14}$.

Une importante jurisprudence s'est alors développée en matière de harcèlement moral au travail. Ainsi, l'employeur est reconnu responsable de l'existence de harcèlement moral au travail même s'il a sanctionné l'auteur des faits ${ }^{15}$. Cependant, le salarié qui a faussement dénoncé des agissements de harcèlement moral pourra être sanctionné jusqu'au licenciement quand la mauvaise foi est prouvée ${ }^{16}$. L'apogée de la jurisprudence en matière de harcèlement moral au travail fut atteint le 10 novembre 2009 à l'occasion de deux arrêts rendus par la chambre sociale de la Cour de cassation qui qualifie pour l'un le harcèlement moral au travail sans intention malveillante de son auteur (« indépendamment de l'intention de son auteur $»)^{17}$ et pour l'autre « des méthodes de gestion mises en œuvre par un supérieur hiérarchique dès lors qu'elles se manifestent pour un salarié déterminé ».

Toutefois, un nouveau tournant a été amorcé avec la décision du 25 novembre 2015 rendu par la Chambre sociale de la Cour de cassation ${ }^{18}$ qui réoriente son approche de l'obligation de sécurité à la charge de l'employeur et qui va impacter l'approche jurisprudentielle du harcèlement au travail. La Chambre sociale a introduit pour la première fois l'article L. 4121-2 relatif aux principes généraux de prévention, qui visent notamment le harcèlement moral au travail, et initie un mouvement de repli qui semble

13 Cass. soc. 28 février 2002, Bull., V, n 81, p. 84 ; M. Babin, N. Pichon N., « Obligation de sécurité et faute inexcusable de l'employeur», Droit social, 2002, p. 828 ; A. Lyon-Caen, "Une révolution dans le droit des accidents du travail », Droit social, 2002, p. 445.

14 Cass. soc. 21 juin 2006, D., 2006, n 41, p. 2831, note M. Miné; RDT, p. 245, obs. P. Adam ; L. Leblanc, « Harcèlement moral. Responsabilité personnelle du salarié et obligation de résultat de l'employeur », RJS, 8-9/06, p.670-675; Ch. Radé, " harcèlement moral et responsabilités au sein de I'entreprise : I'obscur éclaircissement », Droit Social, n 9/10, 2006, p. 825-833.

15 Cass. Soc. 3 février $2010 n^{\circ} 08-40.144$.

16 Cass. Soc. 10 juin $2015 n^{\circ} 14-13.318$ et $13-25.554$.

17 L. Lerouge, "La constitution du harcèlement moral au travail indépendamment de l'intention de son auteur ", note sous Cass. soc. 10 novembre 2009 n $^{\circ}$ 08-41.497, Petites Affiches, $n^{\circ} 28$, 9 février 2010, p. 18-21; L. Lerouge, «Vers la qualification de méthodes de gestion de harcèlement moral ? », note sous Cass. soc. 10 novembre 2009 n07-45.321, Petites Affiches, n²1, 29 janvier 2010, p. 9-12.

18 Cass. Soc. 25 novembre 2015 D. 2015. 2507 ; ibid. 2016. 144, chron. P. Flores, S. Mariette, E. Wurtz et N. Sabotier; v. A. Dejan de la Bâtie, Arrêt Air France : la chambre sociale rend un hommage appuyé à l'obligation de prévention, JSL 7 janv. 2016. 4 ; A. Gardin, La redéfinition de l'obligation de sécurité de l'employeur, RJS 2016. 99 ; M. Babin, L'obligation de sécurité de résultat, nouvelle approche, JCP S 2016. 1011 ; F. Champeaux, L'infléchissement de la jurisprudence sur l'obligation de sécurité, Sem. soc. Lamy 2015, nº 1700 ; JSL 2016, n 401, p. 4, obs. A. Dejean de la Bâtie. 
annoncer l'abandon de la référence à l'obligation de sécurité de résultat en droit du travail. Cette évolution a été confirmée en matière de harcèlement moral par une décision rendue en 2016 le $1^{\text {er }}$ juin 201619 dans laquelle la Cour de cassation reconnait que l'employeur avait pris toutes les mesures nécessaires de prévention au regard des articles L. 4121-1 et 4121-2 du Code du travail, l'exonérant ainsi de sa responsabilité. Toutefois, la non qualification du harcèlement moral par le juge n'exclut pas la responsabilité de l'employeur pour les situations de souffrance au travail20.

Si le contentieux relatif au harcèlement moral a atteint en droit du travail une telle densité, cela s'explique notamment par l'aménagement de la charge de la preuve en faveur de la personne qui se prétend victime de harcèlement moral. En revanche, le contentieux est moins important du côté du droit de la sécurité sociale et en droit pénal en raison de l'absence de ce principe qui rend le harcèlement moral et ses effets plus difficiles à faire reconnaitre, mais n'en reste pas moins intéressant.

\section{II - HARCÈLEMENT MORAL AU TRAVAIL ET JURISPRUDENCE EN DROIT PÉNAL ET DE LA SÉCURITÉ SOCIALE}

La jurisprudence de la sécurité sociale et pénale se distingue de la jurisprudence en droit du travail au regard du régime de la charge de la preuve. En droit de la sécurité sociale, en raison des passerelles avec le droit du travail quand il s'agit d'aborder l'application de la législation professionnelle, les débats se concentrent sur la reconnaissance ou non des effets du harcèlement moral au travail en accident du travail avec plusieurs niveaux de réponse relatifs au lien de causalité $(A)$. En droit pénal en revanche, la réponse est plus tranchée, l'intentionnalité de l'auteur du harcèlement doit être apportée par le demandeur (B).

\section{A - LA JURISPRUDENCE EN DROIT DE LA SÉCURITÉ SOCIALE : LA QUALIFICATION D'ACCIDENT DU TRAVAIL DES EFFETS DU HARCÈLEMENT MORAL}

En droit de la Sécurité sociale, les débats en jurisprudence portent bien sur la reconnaissance des effets sur la santé du harcèlement moral en accident du travail et non pas sur le harcèlement moral lui-même qui ne s'y prête pas par essence. Compte tenu que les troubles de la santé mentale ne sont pas reconnus dans les tableaux de maladies professionnelles et que la reconnaissance par la voie des maladies hors tableau s'avère compliquée, les juges ont généralement rattaché l'application de la législation professionnelles aux accidents du travail qui offre un régime juridique plus commode en l'espèce.

La présomption d'imputabilité vise toute lésion apparue au temps et au lieu de travail constitue un accident imputable au travail ${ }^{21}$. Elle a été créée par la jurisprudence afin d'éviter à la victime d'un accident de prouver son origine professionnelle. Toutefois, cette présomption est une présomption simple qui peut être renversée par la preuve contraire

19 Cass. soc. $1^{\text {er juin } 2016, n^{\circ} 14-19.702 .}$

20 Cass. soc., 6 déc. 2017, n¹6-10.691.

21 Cass. soc. 2 février 1983, Bull. V, nº 71. 
en démontrant que l'origine de l'accident est totalement étrangère au travail ${ }^{22}$. Le 2 avril 2003, la chambre sociale de la Cour de cassation a estimé cependant l'accident du travail pouvait se définir comme « un événement ou une série d'événements survenus à des dates certaines par le fait ou à l'occasion du travail, dont il est résulté une lésion corporelle, quelle que soit la date d'apparition de celle-ci ${ }^{23}$. La définition de l'accident du travail retient donc en 2003 deux critères décisifs qui sont le caractère soudain et le préjudice corporel résultant de l'accident survenu par le fait ou à l'occasion du travail. L'utilisation par les juges du terme "série d'événements " laisse planer la possibilité d'un certain décalage dans le temps, ce que la Cour de cassation accepte en matière de vaccination. Un décalage entre le fait générateur et l'apparition des troubles de la santé est possible ${ }^{24}$. La Cour de cassation a continué d'évoluer en laissant de côté le critère de la "lésion corporelle » à l'occasion de deux affaires relatives à des traumatismes psychologiques post-traumatiques ${ }^{25}$, tout en admettant la réalisation a posteriori de conséquences psychologiques liées à l'accident.

En matière d'accident du travail, la jurisprudence est encore attachée à l'obligation de sécurité de résultat qui a servi en 2002 à travers les arrêts portant sur les affaires amiantes de redéfinir la faute inexcusable de l'employeur ouvrant droit à une réparation complémentaire au titre de l'article L. 452-1 du Code de la sécurité sociale quand l'accident du travail est reconnu. Cette position a été confirmée par un arrêt rendu par l'Assemblée plénière de la Cour de cassation le 24 juin 2005 selon lequel « en vertu du contrat de travail le liant à son salarié, l'employeur est tenu envers celui-ci d'une obligation de sécurité de résultat, notamment en ce qui concerne les accidents du travail ; que le manquement à cette obligation a le caractère d'une faute inexcusable $»^{26}$.

Le comble de cette jurisprudence fut atteint le 22 février 2007. En l'espèce, un mois après un arrêt maladie en raison d'un syndrome anxio-dépressif causé par son travail, un salarié a tenté de se suicider à son domicile. La Cour de cassation conforte la décision des juges du fond de déclarer opposable à l'employeur la décision de la CPAM en estimant qu'un accident qui se produit à un moment où le salarié ne se trouve plus sous la subordination de l'employeur constitue un accident du travail dès lors que le salarié

22 M. Badel, Droit de la Sécurité sociale, Ellipses, Coll. Mise au point, 2006, p. 90 ; Cass. ch. réunies 7 avril 1921, S., 1922, 1, 81.

23 Cass. soc. 2 avril 2003, RJS, 6/03, n 801 ; D., 2003, p. 1724, note K. Gaba ; Dr. soc., 2003, p. 673, obs. L. MILET.

24 Les salariés obligés de se vacciner à la demande de l'employeur et des effets secondaires graves qui se déclarent plusieurs jours après la vaccination, NDLR ; Cass. civ. 2ème 25 mai 2004, RJS, 8-9/04, $n^{\circ} 966$; Cass. civ. $2^{\text {ème }} 22$ mars 2005, RJS, 6/05, nº67 ; D., 2005, p. 2053, note Y. Saint-Jours.

25 À propos d'une dépression consécutive à un entretien d'évaluation : Cass. civ. $2^{2 e ̀ m e} 1^{\text {er }}$ juil. 2003, RJS, 10/03, n 1222 ; D., 2004, p. 906, note M. Huyette ; à propos d'une dépression à la suite d'une agression au travail (attaque à main armée d'une banque) : Cass. civ. $2^{\text {ème }} 15$ juin 2004, RJS, 8-9/04, $n^{\circ} 965$.

26 Cass. Ass. Plén. 24 juin 2005, n 30-30.038; F.-j. Pansier, «Obligation de sécurité de résultat de l'employeur en matière de sécurité ", CSBP, septembre 2005, p. 376 ; A. Dejean de La Bâtie, "Jurisprudence amiante : la consécration », SSL, 2005, p. 1225 ; F. Kessler, « Faute inexcusable : l'Assemblée plénière de la Cour de cassation met fin aux rébellions des juges du fond ", Droit Ouvrier, nov. 2005, p. 473 ; X. Prétot, note sous AP 24 juin 2005, Droit social, 2005, p. 1067 ; O. Pujolar, "Faute inexcusable de l'employeur et faute inexcusable de la victime d'un accident de travail : des confirmations de jurisprudence », Lexbase Hebdo - Edition Sociale, 14 juil. 2005, $n^{\circ} 176$; P.-Y. Verkindt, note sous AP 24 juin 2005, RDSS, 5/2005, p. 875. 
établit qu'il est survenu par le fait du travail. La preuve du lien avec le travail est donc à rapporter du côté du demandeur, mais une situation de harcèlement moral permet de rejeter la faute intentionnelle. Ce lien de causalité est le plus souvent soutenu par des certificats médicaux qui attestent que l'état de santé dans lequel se trouve le salarié résulte de mauvaises conditions de travail. Côté employeur, souvent argumentant que des troubles comme la dépression est avant tout d'origine multifactorielle sans qu'il ne soit possible d'affirmer avec certitude que l'origine est bien professionnelle, le débat s'est porté sur la demande d'une meilleure justification de ce lien de causalité de la part du corps médical. En effet, des médecins sont poursuivis par certains employeurs devant les chambres disciplinaires du conseil de l'ordre en charge de veiller à la déontologie médicale pour justement avoir attesté par un écrit du lien entre la santé et le travail ${ }^{27}$.

Le point commun entre le droit de la sécurité sociale (faute inexcusable) et le droit pénal est le lien avec le travail qui est à démontrer. En revanche, en matière de preuve, l'intentionnalité éloigne le droit de la sécurité sociale du droit pénal.

\section{B - LA JURISPRUDENCE PÉNALE : PROUVER L'INTENTIONNALITÉ DU HARCELEUR PRÉSUMÉ}

Conformément au droit de la procédure pénale, le demandeur à une action pénale en matière de harcèlement moral au travail doit prouver son existence ainsi que l'intentionnalité du harceleur présumé. Les décisions pénales en matière de harcèlement moral au travail ne font pas légion. Le plus souvent, elles résultent d'une mobilisation personnelle d'un inspecteur du travail et d'un(e) Procureur(e) de la République particulièrement conscient des dégâts produits par le harcèlement ${ }^{28}$. Un certain nombre de décisions montrent une absence totale de prise en compte de la personne humaine résultat de décisions de gestion sans aucun discernement ${ }^{29}$.

La lecture de la jurisprudence de la Chambre criminelle de la Cour de cassation montre que sont incriminés pénalement les faits de moqueries, déclassements, humiliations et dénigrement publiques, harcèlement moral discriminatoire en raison de l'exercice d'un mandat syndical, conditions de travail dégradantes (pas de pause, horaires de travail particulièrement étendus, impossibilité de prendre les périodes de repos auxquelles le salarié peut prétendre, sur contrôle des tâches sans justification, ...). La « mise au placard» ou " placardisation » écartant le salarié de relations avec ses collègues et de tâches à réaliser est incriminé par le juge pénal. Ainsi, le 17 juin 2014, la Cour de cassation a rappelé que « la fourniture de travail qui et la contrepartie du salaire perçu est une obligation pour l'employeur qui ne saurait s'en affranchir sans porter atteinte à la dignité du salarié ${ }^{30}$ ».

27 V. Le Monde Économie, «Les médecins veulent pouvoir attester librement les conséquences du travail sur la santé », 19 septembre 2017.

28 Conférence de R. Saada "Politiques pénales en matière de harcèlement moral au travail : quel

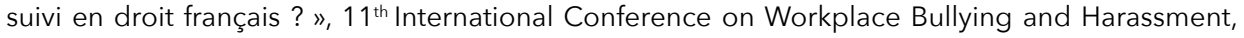
Bordeaux, 6-8 juin 2018.

29 CA Paris, 22 novembre 2011 n $10 / 07194$.

30 Cass. Crim 17 juin $2014 n^{\circ}$ 12-85.869. 
Il arrive par ailleurs que des tensions naissent entre l'incrimination pénale de harcèlement moral au travail après un dépôt de plainte d'un salarié licencié et l'absence de qualification par le juge du travail du licenciement sans motif réel et sérieux. Or, la Chambre sociale de la Cour de cassation a, une nouvelle fois, tranché le 3 février 2017 en retenant que dès lors que le salarié licencié a été victime de harcèlement moral de la part de son supérieur hiérarchique, lequel a été condamné pénalement pour ces faits, son licenciement est nul. Le juge n'a pas à examiner les autres arguments invoqués par l'employeur à l'appui de ce licenciement ${ }^{31}$ en raison du principe de l'autorité de la chose jugée au pénal sur le droit civil. Les juges de cassation rejettent par ailleurs la décision de la Cour d'appel d'accueillir la demande de dommages-intérêts pour harcèlement moral, au motif que la victime avait déjà été indemnisée par le Tribunal correctionnel. La Chambre sociale distingue ici la condamnation pénale par le tribunal correctionnel des dommages subis par la victime et les dommages et intérêts alloués par les juridictions du travail en raison du harcèlement moral résultant des manquements de l'employeur.

Enfin, parmi les décisions marquantes de ce rapide panorama de la jurisprudence pénale, des juridictions sont susceptibles de retenir à l'encontre de l'auteur du harcèlement moral l'incrimination pénale d'homicide involontaire. Il en est ainsi du suicide résultant de conditions de travail mettant tellement à rude épreuve le travailleur, que les méthodes de management à son encontre sont reconnues comme du harcèlement moral ${ }^{32}$. De ce fait, quand le dossier du plaignant ou des ayants droit a été construit sur des preuves tangibles, il pourra aboutir jusqu'à la reconnaissance de l'homicide involontaire, mais à quel prix pour les victimes et les familles! Une action pénale est une nouvelle épreuve, longue et tellement éprouvante. L'enjeu est donc bien celui de la prévention pensée pour enrayer tant de gâchis. 